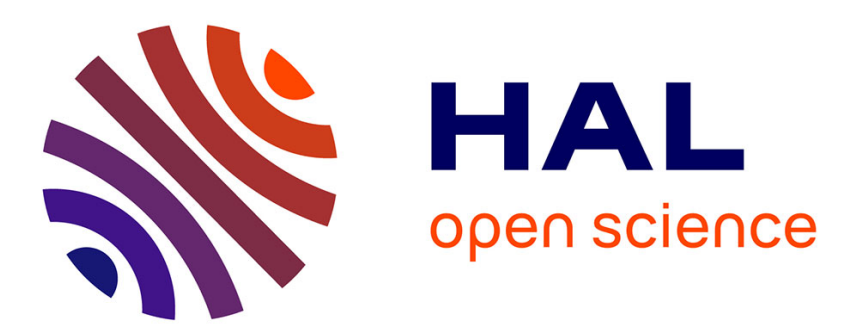

\title{
Adaptive leader-follower formation control of mobile robots following arbitrary reference trajectories
}

\author{
Mohamed Maghenem, Antonio Loria, Elena Panteley
}

\section{To cite this version:}

Mohamed Maghenem, Antonio Loria, Elena Panteley. Adaptive leader-follower formation control of mobile robots following arbitrary reference trajectories. 23rd International Conference on System Theory, Control and Computing (ICSTCC 2019), Oct 2019, Sinaia, Romania. 10.1109/ICSTCC.2019.8885584 . hal-02367899

\section{HAL Id: hal-02367899 \\ https://hal.science/hal-02367899}

Submitted on 5 Mar 2020

HAL is a multi-disciplinary open access archive for the deposit and dissemination of scientific research documents, whether they are published or not. The documents may come from teaching and research institutions in France or abroad, or from public or private research centers.
L'archive ouverte pluridisciplinaire HAL, est destinée au dépôt et à la diffusion de documents scientifiques de niveau recherche, publiés ou non, émanant des établissements d'enseignement et de recherche français ou étrangers, des laboratoires publics ou privés. 


\title{
Adaptive leader-follower formation control of mobile robots following arbitrary reference trajectories
}

\author{
Mohamed Maghenem Antonio Loría Elena Panteley *†
}

\begin{abstract}
We address the problem of leader-follower formation control of nonholonomic vehicles with parametric uncertainty. The controller that we propose applies to diverse kinds of reference trajectories, including set-point stabilisation, persistently-exciting trajectories, and converging ones and ensures uniform global asymptotic stability in closed loop. In particular, for the first time in the literature, we establish uniform convergence of the parameter estimation errors in such context. Also, some original simulation results illustrate our theoretical findings.
\end{abstract}

Index terms - Formation control, persistency of excitation, Lyapunov design, nonholonomic systems

\section{INTRODUCTION}

Tracking and set-point stabilisation control of nonholonomic vehicles is a topic that has not ceased to attract the regard of the control community even if, in the interval of a few decades, a tremendous bulk of literature on the subject has already been produced — see, e.g., [1]-[4]. Contemporary problems such as formation control of autonomous swarms of vehicles, consensus control, and other scenarios of cooperative systems communicating over networks maintain the interest for the basic tracking and set-point stabilisation control of nonholonomic systems .

From an academic viewpoint, these systems are attractive because their analysis imposes considerable challenges depending on the nature of the reference trajectories. Roughly speaking, these may be rich in a persistency-of-excitation ([5]) sense, as e.g., in [6], [7], they can be "converging", as in [3] and [8]-[10] or, simply, correspond to a set-point [2]. Most often, the controllers designed for a type of reference trajectory does not apply for another; designing a universal controller that applies indistinctly to the case of persistent or converging trajectories is a very challenging problem that has been little addressed in the literature. In the case of one leader and one follower it has been studied in [8] and in [10]-[14].

In [8] several scenarios of tracking control (circular paths, straight-line paths, vanishing trajectories) are covered. In [10] it is assumed that the reference trajectory is either persistently exciting or integrable - note that integrability imposes certain speed of convergence. In both references, however, it is assumed that the vehicles are velocity-controlled (that

\footnotetext{
$\dagger$ Research funded in part by ANR via project HANDY, number ANR-18CE40-0010 and by the Government of Russian Federation (grant 074-U01).

* M. Maghenem is with Univ of California, Santa Cruz, CA, USA. E-mail: mmaghene@ucsc.edu. A. Loria and E. Panteley are with LSSCentraleSupelec, CNRS, 3 Rue Joliot Curie, 91192, France. E-mail: loria(panteley)@1ss.supelec.fr. E. Panteley is also with ITMO University, Kronverkskiy av. 49, Saint Petersburg, 197101, Russia.
}

is, only the kinematics model is considered). The framework laid in [12] is very general as it applies to chainform systems, which is a class that includes the unicycle model, most often used. "Full" models of torque-controlled vehicles, that is, which include the Lagrangian dynamics, are considered in [13] and [14]. In the former the convergence of the error positions to a steady-state error, albeit under parametric uncertainty, is established. The convergence to zero of the same errors is guaranteed by the controller reported in [14] provided that either the forward reference velocity is separated from zero (whence persistently exciting) or the angular reference velocity is separated from zero and the forward velocity is integrable in norm. In [11] a robust controller that guarantees the stronger properties of uniform global asymptotic stability and integral input to state stability for the kinematics closed-loop equation is proposed.

The simultaneous tracking and robust stabilisation control problem for groups of robots $(N>2)$, has been addressed in [15]-[17]. In [15] it is established that the formationerrors converge to an arbitrarily small compact ball centred at the origin; the controller is centralised hence, it is assumed that the leader's velocities are accessible to all the agents in the network. In [16] and [17] it is established, for the first time, uniform global asymptotic stability for the closed-loop system. The main results in this paper, build upon the latter two references to address the problem of adaptive control and closed-loop identification. We consider vehicles modelled by a unicycle kinematics and a Lagrangian-dynamics equation and we suppose that the lumped parameters in the latter are unknown. Under these conditions, we establish, for the first time in the literature, uniform global asymptotic stability for the closed-loop system of a swarm of nonholonomic vehicles in closed loop with a unique controller that is capable of stabilising both converging and exciting trajectories. From a technical viewpoint, we also emphasise that the main statement implies uniform parametric error convergence.

The rest of the paper is organised as follows. In the next section we recall the equations of the unicycle model and formulate the control problem. In Section III we present, without proof, some statements that appear in [17]. In Section IV we present our main results. Original simulation tests that illustrate our theoretical findings are provided in Section V and we conclude with some remarks in Section VI.

\section{MODEL AND PROBLEM FORMULATION}

Consider $N$ nonholonomic vehicles moving on the plane, with kinematics modelled by the unicycle equations

$$
\dot{x}_{i}=v_{i} \cos \left(\theta_{i}\right)
$$




$$
\begin{aligned}
\dot{y}_{i} & =v_{i} \sin \left(\theta_{i}\right) \\
\dot{\theta}_{i} & =\omega_{i} \quad i \in\{1, \ldots N\}
\end{aligned}
$$

where $x_{i} \in \mathbb{R}$ and $y_{i} \in \mathbb{R}$ correspond to the Cartesian coordinates of a point on the $i$ th vehicle with respect to a fixed reference frame and $\theta_{i} \in \mathbb{R}$ denotes the vehicle's orientation. In turn, the forward velocity $v_{i}=\left[\dot{x}_{i}+\dot{y}_{i}\right]^{1 / 2}$ and the angular velocity $\omega_{i}$ result from the Lagrangian equations

$$
\begin{aligned}
& M_{i} \dot{\eta}_{i}+C_{i}\left(\eta_{i}\right) \eta_{i}=u_{i}, \\
& \eta_{i}:=\left[\begin{array}{ll}
v_{i} & \omega_{i}
\end{array}\right]^{\top},
\end{aligned}
$$

where $M_{i}$ and $C_{i}$ are, respectively, the inertia and the Coriolis-and-centrifugal-forces matrices, which are given by

$$
M_{i}=\left[\begin{array}{ll}
m_{i 1} & m_{i 2} \\
m_{i 2} & m_{i 1}
\end{array}\right], \quad C_{i}\left(\eta_{i}\right)=\left[\begin{array}{cc}
0 & c_{i} \omega_{i} \\
-c_{i} \omega_{i} & 0
\end{array}\right],
$$

and the control inputs are $u_{i}:=B_{i} \tau_{i}$ where, in turn, $B_{i} \in$ $\mathbb{R}^{2 \times 2}$ is a full rank constant matrix of known coefficients and $\tau_{i}$ is the vector of input torques at the wheels $-c f$. [3].

Let such group of vehicles communicate in a leaderfollower fashion that is, assume that for each $i \leq N$, the $i$ th robot receives the states of exactly one leader, labelled $(i-1)$; the $N$ th vehicle having no follower and the leader to the first vehicle being a fictitious unicycle with kinematics

$$
\begin{aligned}
& \dot{x}_{r}=v_{r} \cos \left(\theta_{r}\right) \\
& \dot{y}_{r}=v_{r} \sin \left(\theta_{r}\right) \\
& \dot{\theta}_{r}=\omega_{r} .
\end{aligned}
$$

The leader-follower formation control problem consists in the vehicles acquiring and maintaining a specified physical formation relative to one another and following reference trajectories generated by a fictitious robot. From a controltheory viewpoint, this can be stated as a stabilisation problem as follows.

Let $v_{r}, \omega_{r}$ be given piece-wise continuous functions mapping $\mathbb{R}_{>0} \rightarrow \mathbb{R}$ that represent the forward and angular reference velocities respectively and let $z_{r}:=\left[x_{r}, y_{r}, \theta_{r}\right]^{\top}$ denote the position and orientation reference coordinates which result from (4). A given relative formation may be designed by imposing certain desired Cartesian distances $d_{x i}$ and $d_{y i}>0$ between each leader-follower couple, that is,

$$
\begin{aligned}
& p_{\theta i}:=\theta_{i-1}-\theta_{i}, \\
& p_{x i}:=x_{i-1}-x_{i}-d_{x i}, \\
& p_{y i}:=y_{i-1}-y_{i}-d_{y i} .
\end{aligned}
$$

Then, transforming the error coordinates $\left(p_{\theta}, p_{x}, p_{y}\right)$ of the leader vehicle from the global coordinate frame to local coordinates fixed on the vehicle, we define $e_{i}:=\left[e_{\theta i} e_{x i} e_{y i}\right]^{\top}$,

$$
\left[\begin{array}{l}
e_{\theta i} \\
e_{x i} \\
e_{y i}
\end{array}\right]:=\left[\begin{array}{ccc}
1 & 0 & 0 \\
0 & \cos \left(\theta_{i}\right) & \sin \left(\theta_{i}\right) \\
0 & -\sin \left(\theta_{i}\right) & \cos \left(\theta_{i}\right)
\end{array}\right]\left[\begin{array}{l}
p_{\theta i} \\
p_{x i} \\
p_{y i}
\end{array}\right],
$$

which satisfy

$$
\begin{aligned}
\dot{e}_{\theta i} & =\omega_{i-1}-\omega_{i} \\
\dot{e}_{x i} & =\omega_{i} e_{y i}-v_{i}+v_{i-1} \cos \left(e_{\theta i}\right)
\end{aligned}
$$

$$
\dot{e}_{y i}=-\omega_{i} e_{x i}+v_{i-1} \sin \left(e_{\theta i}\right),
$$

where $v_{i-1}$ and $\omega_{i-1}$ are, respectively, the forward and angular velocities of the leader vehicle. In (6) we set $v_{0}:=v_{r}$ and $\omega_{0}:=\omega_{r}$ where $v_{r}$ and $\omega_{r}$.

Thus, the leader-follower formation control problem consists in designing control inputs $u_{i}:=\left[\begin{array}{ll}u_{i 1} & u_{i 2}\end{array}\right]^{\top}$, with $i \in\{1 \ldots n\}$, such that

$$
\lim _{t \rightarrow \infty} e_{i}(t)=0 \quad \forall i \in\{1 \ldots N\}
$$

hold for the system (1)-(2a).

From a control-theory perspective, however, having (7) as objective is somewhat under-challenging since it does not comprise any stability nor uniformity properties. Instead, we solve the following problem. Let $\hat{\Theta}_{i}$ be the estimate of $\Theta_{i}:=\left[\begin{array}{lll}m_{i 1} & m_{i 2} & c_{i}\end{array}\right]^{\top}$ and let $\tilde{\Theta}_{i}:=\hat{\Theta}_{i}-\Theta_{i}$. Given a piecewise continuous function $\mathbb{R}_{\geq 0} \rightarrow \mathbb{R}^{2}, \eta_{r}:=\left[\begin{array}{ll}v_{r} & \omega_{r}\end{array}\right]^{\top}$ that generates, through (4), feasible trajectories $t \mapsto z_{r}$, design virtual control laws $v_{i}^{*}$ and $\omega_{i}^{*}$ and a control law $u_{i}$ such that, defining,

$$
\tilde{v}_{i}:=v_{i}-v_{i}^{*}, \quad \tilde{\omega}_{i}:=\omega_{i}-\omega_{i}^{*}, \quad \text { and } \quad \tilde{\eta}_{i}:=\left[\begin{array}{ll}
\tilde{v}_{i} & \tilde{\omega}_{i}
\end{array}\right]^{\top},
$$

the origin for the closed-loop system, $\left\{\left(e_{i}, \tilde{\eta}_{i}, \tilde{\Theta}_{i}\right)=\right.$ $(0,0,0)\}$ is uniformly globally asymptotically stable.

Such leader-follower formation control problem for arbitrary feasible reference trajectories is beyond reach, even in the one-leader-one-follower scenario [18]. In this paper we address it for a fairly general class of references that includes set-points, vanishing trajectories (so-called robust stabilisation), and persistently-exciting trajectories. More precisely, we assume that reference velocities satisfy either of the following mutually exclusive conditions:

$$
\int_{t}^{t+T}\left|\eta_{r}(\tau)\right|^{2} d \tau \geq \mu \quad \forall t \geq 0
$$

The main contribution of this paper is a unique adaptive controller that ensures uniform global asymptotic stability in the space of the closed-loop system and, in the case that the lumped parameters of $M$ and $C$ are unknown, the property continues to hold, provided that the reference trajectories satisfy (9a). Note that this includes the uniform convergence of the parameter estimation errors.

\section{LEADER-FOLLOWER FORMATION CONTROL}

The rationale of the control scheme consists in applying recursively an adaptive controller for the one-leader-onefollower scenario that is, it relies on the premise that leaderfollower formation control may be regarded as a cascade of successive master-slave couples. Then, for each robot the controller is designed following a basic backstepping procedure. First, virtual control laws $v_{i}^{*}$ and $\omega_{i}^{*}$ are defined for the unicycle model in error coordinates, (6). Then, a tracking controller at the dynamics-equations level, (2), is designed so that $\eta_{i} \rightarrow \eta_{i-1}$, even in the case of parametric uncertainty. For each vehicle, the control $u_{i}$ depends on the $i$ th vehicle's state-variables and its leader's only. For clarity 
of exposition, we present first the solution for one arbitrary pair of robots, at the kinematic level $\_c f$. [11].

\section{A. One-leader-one-follower tracking control}

Consider an arbitrary pair of leader-follower vehicles, labelled $i$ and $i-1$, for which (7) must hold. We define

$$
\begin{aligned}
v_{i}^{*}= & v_{i-1} \cos \left(e_{\theta i}\right)+k_{x i} e_{x i} \\
\omega_{i}^{*}= & \omega_{i-1}+k_{\theta i} e_{\theta i}+k_{y i} e_{y i} v_{i-1} \phi\left(e_{\theta i}\right) \\
& \quad+\rho_{i}(t) k_{y i} p_{i}(t)\left|e_{x y i}\right|
\end{aligned}
$$

where $e_{x y i}:=\left[\begin{array}{ll}e_{x i} & e_{y i}\end{array}\right]^{\top}$, the function $p_{i}: \mathbb{R}_{\geq 0} \rightarrow \mathbb{R}_{\geq 0}$ is assumed to be once continuously differentiable, bounded, and with bounded derivative $\dot{p}_{i}, \phi: \mathbb{R}_{\geq 0} \rightarrow \mathbb{R}_{\geq 0}$ is the socalled $\operatorname{sinc}(\cdot)$ function,

$$
\phi(x)=\frac{\sin (x)}{x}
$$

and $k_{x i}, k_{y i}, k_{\theta i}$ are positive constants.

Roughly speaking, akin to [10], the controller is constructed as a combination of two control laws; one that ensures tracking of rich trajectories satisfying (9a) and another one that ensures tracking of vanishing ones, satisfying (9b). More precisely, the first three terms on the right-hand side of (10b) guarantee the achievement of the tracking control goal of persistently-exciting trajectories, while the fourth one ensures tracking of converging trajectories. Then, the function $\rho_{i}$ is designed to smoothly "weigh" the effect of one term or the others relatively to the trajectory to be tracked: $\rho_{i}$ is required to be approximately null (thereby enforcing the action of the third term in (10b)) to track persistently exciting trajectories and it is required to remain separated from zero to favour the tracking control of vanishing trajectories. To that end, we define $\rho_{i}: \mathbb{R}_{\geq 0} \rightarrow \mathbb{R}_{\geq 0}$ as the solution of

$$
\dot{\rho}_{i}:=-F\left(\eta_{i-1}(t)\right) \rho_{i}
$$

where $F: \mathbb{R}^{2} \rightarrow \mathbb{R}_{\geq 0}$ is a piece-wise continuous function defined as

$$
F(\eta):=\left\{\begin{array}{cl}
0 & \text { if } \eta \in\left(0, \frac{\mu}{2 T \bar{\eta}}\right] \\
\alpha(|\eta|) & \text { otherwise }
\end{array}\right.
$$

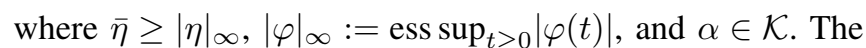
interest this definition is that $F\left(\eta_{r}(\cdot)\right)$ is persistently exciting, i.e., there exist $\mu_{1}$ and $T_{1}>0$ such that

$$
\int_{t}^{t+T_{1}} F\left(\eta_{r}(s)\right)^{2} d s \geq \mu_{1}, \quad \forall t \geq 0
$$

under (9a) and $F\left(\eta_{r}(\cdot)\right)$ is integrable, i.e.,

$$
\int_{0}^{\infty} F\left(\eta_{r}(s)\right) d s \leq \beta,
$$

under (9b).

Under these conditions, we have the following statement on the convergence of the tracking errors.
Proposition 1 Let $i \leq N$ be arbitrarily fixed and consider the system (6) with state $e_{i}$, exogenous signal $\eta_{i-1}=$ $\left[v_{i-1}, \omega_{i-1}\right]$ such that

$$
\max \left\{\left|\eta_{i-1}\right|_{\infty},\left|\dot{\eta}_{i-1}\right|_{\infty}\right\} \leq \bar{\eta}_{i-1},
$$

and inputs $\omega_{i}$ and $v_{i}$. Consider the virtual control laws $\left(v_{i}^{*}, \omega_{i}^{*}\right)$ as given by (10), (11)-(14), with the functions $p_{i}$ and $\dot{p}_{i}$ being bounded and persistently exciting. Then, if $\tilde{v}_{i}:=v_{i}-v_{i-1}$ and $\tilde{\omega}_{i}:=\omega_{i}-\omega_{i-1}$ are bounded, the trajectories exist on $\left[t_{0}, \infty\right)$. Furthermore,

1) if (9a) holds with $\eta_{r}$ replaced by $\eta_{i-1}$, the system is integral input-to-state stable with respect to the input $\tilde{\eta}_{i}$. Consequently, if $\tilde{\eta}_{i}$ tends to zero and is square integrable, the limit in (7) holds.

2) If, alternatively, (9b) holds with $\eta_{r}$ replaced by $\eta_{i-1}$ the system is small input-to-state stable with respect to the input $\tilde{\eta}_{i}$ and if $\tilde{\eta}_{i}$ converges to zero the limit in (7) holds.

The proof is omitted here due to space constraints; interested readers may see [16]. The statement in Proposition 1 is significant for several reasons. Firstly, it establishes small and integral input-to-state stability for the closed-loop system; these are robustness properties that allow to consider the kinematics and dynamics control loops separately, that is as two systems in cascade $-c f$. [19]. In turn, it may be established that if not only $\tilde{\eta}_{i} \rightarrow 0$, but $\left\{\tilde{\eta}_{i}=0\right\}$ is UGAS, then, the origin for the overall closed-loop system is also UGAS - see Proposition 2 below. Finally, the robustness statement of Proposition 1 allows to extend this result to the case of successive pairs of leader-follower couples, that is, to solve the leader-follower formation-and-tracking control problem. This is presented in the following section.

Proposition 2 (UGAS of the full model) Consider the system (1), (2) under the action of any controller $u_{i}$ guaranteeing uniform global asymptotic stability of $\left\{\tilde{\eta}_{i}=0\right\}$ and that $\tilde{\eta}_{i} \in \mathcal{L}_{2}$. Then, under the conditions of Proposition 1 , the origin $\left(\tilde{e}_{i}, \tilde{\eta}_{i}\right)=(0,0)$ is uniformly globally asymptotically stable.

The statement of Proposition 2 relies on a cascaded-systems stability argument. Let $u_{i}$ be a given controller for the dynamics equations (2), depending on the leader and follower's states, as well as on the virtual control laws (10). Then, by a suitable change of coordinates the closed-loop equations take the generic form

$$
\dot{\tilde{\eta}}_{i}=F_{\eta i}\left(t, \tilde{\eta}_{i}, e_{i}\right) \text {. }
$$

Next, we replace $e_{i}$ in (16) by complete trajectories $e_{i}(t)$ so the overall closed-loop equations cover a cascaded form

$$
\begin{aligned}
& \dot{e}_{i}=F_{e i}\left(t, e_{i}\right)+G_{e i}\left(t, e_{i}\right) \tilde{\eta}_{i} \\
& \dot{\tilde{\eta}}_{i}=\tilde{F}_{\eta i}\left(t, \tilde{\eta}_{i}\right)
\end{aligned}
$$

where $\tilde{F}_{\eta i}\left(t, \tilde{\eta}_{i}\right):=F_{\eta i}\left(t, \tilde{\eta}_{i}, e_{i}(t)\right)-c f$. [19], [20, p. 627]. Equation (17) is a compact representation of Equations (6).

Now, after Proposition 1, if (9a) holds the system (17) is integral-input-to-state stable while, if (9b) holds it is 
small input-to-state stable. On the other hand, either of these conditions implies the so-called 0-UGAS property, that is, uniform global asymptotic stability for $\dot{e}_{i}=F_{e i}\left(t, e_{i}\right)$. On the other hand, the origin for (18) is also UGAS. Thus, after [21, Lemma 2], the origin $\left(e_{i}, \tilde{\eta}_{i}\right)=(0,0)$ is uniformly globally asymptotically stable if the solutions of (17) are uniformly globally bounded. The latter follows under condition (9a), from the integral-input-to-state-stability property and the assumption that $\tilde{\eta}_{i} \in \mathcal{L}_{2}$ in the case of persistentlyexciting reference trajectories and under condition (9b) and the property of small input-to-state stability (see Proposition 1) in the case of vanishing trajectories.

\section{B. Leader-follower formation tracking control}

We consider next a network of autonomous vehicles $(N \geq$ 2 ) that communicate according to a spanning-tree graph and which are required to follow a reference fictitious vehicle following reference trajectories that satisfy either (9a) or (9b). We have the following.

Proposition 3 Consider the system (1), (2). Let $\eta_{r}=$ $\left[v_{r} \omega_{r}\right]^{\top}$ be a given piece-wise continuous function satisfying either (9a) or (9b) and assume that there exists $\bar{\eta}_{r}>0$ such that

$$
\max \left\{\left|\eta_{r}\right|_{\infty},\left|\dot{\eta}_{r}\right|_{\infty}\right\} \leq \bar{\eta}_{r}
$$

For each $i \leq N$ consider the expressions of $v_{i}^{*}$ and $\omega_{i}^{*}$ as in (10) (with $v_{0}:=v_{r}$ and $\omega_{0}:=\omega_{r}$ ) where:

(i) $k_{x i}, k_{y i}, k_{\theta i}$ are positive constants;

(ii) the functions $p_{i}$ and $\dot{p}_{i}$ are bounded and persistently exciting.

Then, for any given control laws $u_{i 1}$ and $u_{i 2}$ guaranteeing that $\tilde{\eta}_{i}$ is square integrable and converges to zero, the control objective (7) holds.

Furthermore, define $\tilde{\eta}:=\left[\tilde{\eta}_{1} \cdots \tilde{\eta}_{N}\right]^{\top}, \quad \eta^{*}:=$ $\left[\eta_{1}^{*} \cdots \eta_{N}^{*}\right]^{\top}$, and $e:=\left[e_{1} \cdots e_{N}\right]^{\top}$. If $\{\tilde{\eta}=0\}$ for (18) is uniformly globally asymptotically stable (UGAS) and $\tilde{\eta} \in \mathcal{L}_{2}$ then, for the closed-loop system (17)-(18), $\{(e, \tilde{\eta})=(0,0)\}$ is also UGAS. Consequently, if $\tilde{\eta} \equiv 0$ then $\{e=0\}$ for (1) in closed loop with $\eta^{*}$ is UGAS.

The proof of this statement consists in applying recursively the statement of Proposition 1 for each $i \leq N$ that is, for each pair of leader-follower vehicles. Indeed, Proposition 1 guarantees the asymptotic convergence of the formation errors whether the leader velocities are persistently exciting or converging. Therefore, the properties of $(i-1)$ th leader's velocities are propagated to the $i$ th follower and, in turn, to the $(i+1)$ th vehicle down to the leaf nodes in the graph.

We use $\omega_{i}=\tilde{\omega}_{i}+\omega_{i}^{*}$ and $v_{i}=\tilde{v}_{i}+v_{i}^{*}$ in (6), together with (10) to write the error-dynamics equations as

$$
\dot{e}_{i}=A_{v_{i-1}}\left(t, e_{i}\right) e_{i}+B_{1 i}\left(t, e_{i}\right) \rho_{i}(t)+B_{2 i}\left(e_{i}\right) \tilde{\eta}_{i},
$$

where

$$
A_{v_{i-1}}:=\left[\begin{array}{ccc}
-k_{\theta i} & 0 & -v_{i-1}(t) k_{y i} \phi\left(e_{\theta i}\right) \\
0 & -k_{x i} & \varphi_{i}\left(t, e_{i}\right) \\
v_{i-1}(t) \phi\left(e_{\theta i}\right) & -\varphi_{i}\left(t, e_{i}\right) & 0
\end{array}\right],
$$

$$
B_{1 i}:=\left[\begin{array}{c}
-k_{y i} p_{i}(t)\left|e_{x y i}\right| \\
k_{y i} p_{i}(t)\left|e_{x y i}\right| e_{y i} \\
-k_{y i} p_{i}(t)\left|e_{x y i}\right| e_{x i}
\end{array}\right], \quad B_{2 i}:=\left[\begin{array}{cc}
0 & -1 \\
-1 & e_{y i} \\
0 & -e_{x i}
\end{array}\right]
$$

and $\varphi_{i}\left(t, e_{i}\right):=\omega_{i-1}+k_{\theta i} e_{\theta i}+k_{y i} e_{y i} v_{i-1} \phi\left(e_{\theta i}\right)$. We stress that these closed-loop equations have the convenient triangular structure

$$
\begin{aligned}
& \dot{e}_{N}=A_{v_{N-1}}\left(t, e_{N}\right) e_{N}+B_{1 N}\left(t, e_{N}\right) \rho_{N}+B_{2 N}\left(e_{N}\right) \tilde{\eta}_{N} \\
& \text { : } \\
& \dot{e}_{2}=A_{v_{1}}\left(t, e_{2}\right) e_{2}+B_{12}\left(t, e_{2}\right) \rho_{2}+B_{22}\left(e_{2}\right) \tilde{\eta}_{2} \\
& \dot{e}_{1}=A_{v_{r}}\left(t, e_{1}\right) e_{1}+B_{11}\left(t, e_{1}\right) \rho_{1}+B_{21}\left(e_{1}\right) \tilde{\eta}_{1}
\end{aligned}
$$

Note that for the $i$ th vehicle the dynamics equations depend on $e_{i}$ and, through $\eta_{i-1}=\left[\begin{array}{ll}v_{i-1} & \omega_{i-1}\end{array}\right]^{\top}$, on the states of the vehicles above in the graph, up to the reference vehicle. However, in view of forward completeness (which can be established as in the proof of Proposition 1), for the purpose of analysis the velocities $\eta_{i-1}$ may be regarded as exogenous signals. This allows us to consider the system as a multicascaded time-varying one — see [19]. Then, we may invoke Proposition 1 recursively.

\section{MAIN RESULTS}

The previous propositions, whose proofs may be found in [16], establish uniform global asymptotic stability for the unicycle kinematics and set the basis for the following statements, which is the first of its kind in the literature.

Proposition 4 Consider the the system (1), (2) in closed loop with (10) and

$$
u_{i}=M_{i} \dot{\eta}_{i}^{*}+C_{i}\left(\eta_{i}\right) \eta_{i}^{*}-k_{d i} \tilde{\eta}_{i}, \quad k_{d i}>0 .
$$

Let condition (19) as well as items (i) and (ii) of Proposition 3 hold. Then, the origin in the state space of the closed-loop system is uniformly globally asymptotically stable.

Proof: The closed-loop dynamics (16) is

$$
M_{i} \dot{\tilde{\eta}}_{i}+C_{i}\left(\tilde{\eta}_{i}+\eta_{i}^{*}\left(t, e_{i}\right)\right) \tilde{\eta}_{i}+k_{d i} \tilde{\eta}_{i}=0, \quad i \leq N
$$

which may be rewritten along complete solutions $e_{i}(t)$ in the form (18). Then, after the skew-symmetry of $C_{i}(\cdot)$, we have

$$
V\left(\tilde{\eta}_{i}\right):=\tilde{\eta}_{i}^{\top} M \tilde{\eta}_{i} \Longrightarrow \dot{V}\left(\tilde{\eta}_{i}\right)=-2 k_{d i}\left|\tilde{\eta}_{i}\right|^{2},
$$

so $\{\tilde{\eta}=0\}$ is a uniformly (in the initial times $t_{\circ}$ and in the trajectories $\left.e_{i}(t)\right)$ globally exponentially stable equilibrium of (23). The result follows from Proposition 3.

Let us now assume that the constant lumped parameters in $M_{i}$ and $C_{i}\left(\eta_{i}\right)$, denoted $\Theta_{i} \in \mathbb{R}^{m}$, are unknown and let $\hat{M}_{i}$ and $\hat{C}_{i}$ denote the estimates of the inertia and Coriolis matrices respectively. Let $\hat{\Theta}_{i}$ correspond to an estimate of $\Theta_{i}$ and consider the controller

$$
\begin{aligned}
u_{i} & =\hat{M}_{i} \dot{\eta}_{i}^{*}+\hat{C}_{i}\left(\eta_{i}\right) \eta_{i}^{*}-k_{d i} \tilde{\eta}_{i}, \quad k_{d i}>0 \\
\dot{\hat{\Theta}}_{i} & =-\gamma \Phi_{i}\left(t, \dot{\eta}_{i}^{*}, \eta_{i}^{*}, \tilde{\eta}_{i}\right)^{\top} \tilde{\eta}_{i}, \quad \gamma>0
\end{aligned}
$$

where, for any $i \leq N, \Phi_{i}$ is a smooth function implicitly defined by the expression

$$
\Phi_{i}\left(t, \dot{\eta}_{i}^{*}, \eta_{i}^{*}, \tilde{\eta}_{i}\right) \tilde{\Theta}_{i}:=\left[\hat{C}_{i}-C_{i}\right] \eta_{i}^{*}+\left[\hat{M}_{i}-M_{i}\right] \dot{\eta}_{i}^{*}
$$


where $\left[\hat{C}_{i}-C_{i}\right]$ is a function of $\eta_{i}=\tilde{\eta}_{i}+\eta_{i}^{*}$.

Proposition 5 Consider the system (1), (2) in closed loop with (10) and (24). Then, the origin $\left\{\left(e_{i}, \tilde{\eta}_{i}, \tilde{\Theta}_{i}\right)=(0,0,0)\right\}$, for all $i \leq N$, is a uniformly globally asymptotically stable equilibrium point if $\Phi_{1}\left(\cdot, \dot{\eta}_{r}(\cdot), \eta_{r}(\cdot), 0\right)$ is persistently exciting.

Proof: The closed-loop system corresponding to the force equations (2) is

$$
\begin{aligned}
& M_{i} \dot{\tilde{\eta}}_{i}+C_{i}\left(\eta_{i}\right) \tilde{\eta}_{i}+k_{d i} \tilde{\eta}_{i}=\Phi_{i}\left(t, \dot{\eta}_{i}^{*}, \eta_{i}^{*}, \tilde{\eta}_{i}\right) \tilde{\Theta}_{i} \\
& \dot{\tilde{\Theta}}_{i}=-\gamma \Phi_{i}\left(t, \dot{\eta}_{i}^{*}, \eta_{i}^{*}, \tilde{\eta}_{i}\right)^{\top} \tilde{\eta}_{i} .
\end{aligned}
$$

In view of (the proof of) Proposition 4, uniform global asymptotic stability of the origin $\left(\tilde{\eta}_{i}, \tilde{\Theta}_{i}\right)$ for (26) follows directly from [22, Theorem 3], provided that $\Phi_{i}\left(\cdot, \dot{\eta}_{i}^{*}(\cdot), \eta_{i}^{*}(\cdot), 0\right)$ is persistently exciting. Now, for $i=1$, this means that $\Phi_{1}\left(\cdot, \dot{\eta}_{r}(\cdot), \eta_{r}(\cdot), 0\right)$ must be persistently exciting, which holds by assumption. We conclude that $\eta_{1} \rightarrow \eta_{r}$ and $\dot{\eta}_{1} \rightarrow \dot{\eta}_{r}$ hence, $\Phi_{2}\left(\cdot, \dot{\eta}_{1}(\cdot), \eta_{1}(\cdot), 0\right)$ is also persistently exciting. The result follows by induction.

\section{Simulation Results}

For the sake of illustration, we have performed some numerical simulations using Simulink of Matlab. We consider a group of four mobile robots required to follow a virtual leader while assuming a diamond-shape formation, which is designed by imposing desired distances between the robots as follows: $\left[d_{x_{r, 1}}, d_{y_{r, 1}}\right]=[0,0],\left[d_{x_{1,2}}, d_{y_{1,2}}\right]=[-1,0]$ and $\left[d_{x_{2,3}}, d_{y_{2,3}}\right]=[1 / 2,-1 / 2]$ and $\left[d_{x_{3,4}}, d_{y_{3,4}}\right]=[0,1]$. Each vehicle is considered to be modelled by (1)-(3) with $m_{i 1}=0.6227, m_{i 2}=-0.2577$, and $c_{i}=0.2025$. The control gains are set to $k_{x_{i}}=k_{y_{i}}=k_{\theta_{i}}=1$, and $k_{d}=15$ while $p(t):=20 \sin (0.5 t)$, which has a persistently exciting time-derivative. The results of two simulation tests are presented. In the first case, the reference vehicle trajectories are generated by (4) with $v_{r}(t)$ and $\omega_{r}(t)$ such that $\left|\eta_{r}(t)\right|$ is persistently exciting - see Figure 1 . That is, in the first simulation test, the reference trajectories satisfy Inequality (9a) and so does the function

$$
F\left(\eta_{r}\right):=\left\{\begin{array}{cc}
0 & \text { if } \eta_{r} \in(0,0.1] \\
\left|\eta_{r}\right| & \text { otherwise. }
\end{array}\right.
$$

The position and velocity tracking errors, in norm, are shown in Figures 2 and 3 respectively.

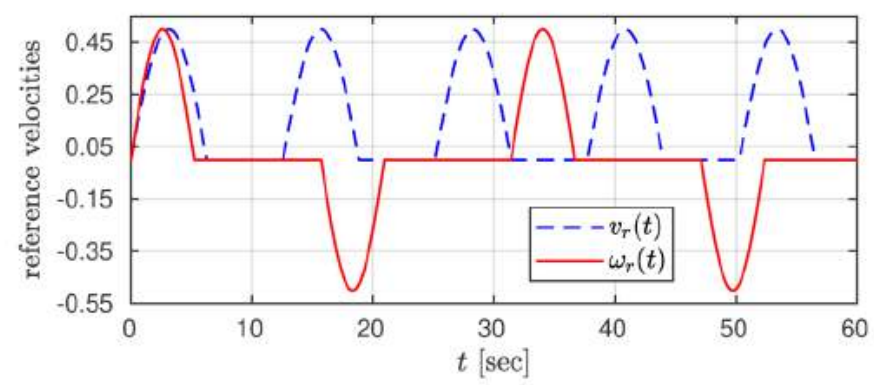

Fig. 1. Reference velocity trajectories $v_{r}(t)$ and $\omega_{r}(t)$

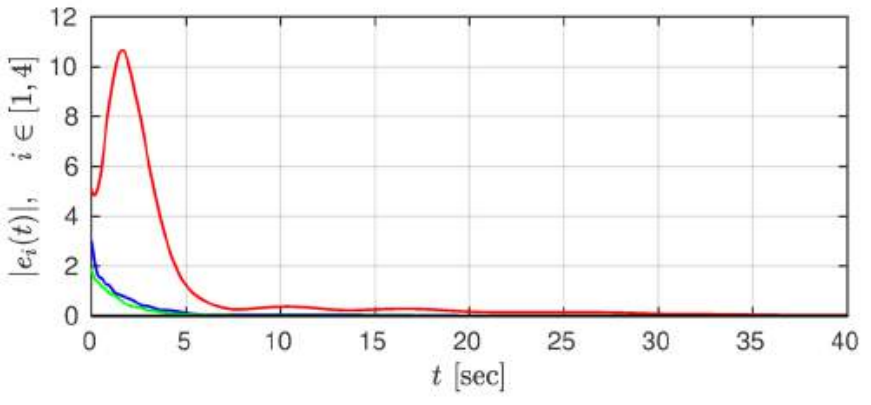

Fig. 2. Normed relative errors for each pair leader-follower, with persistently exciting reference trajectories

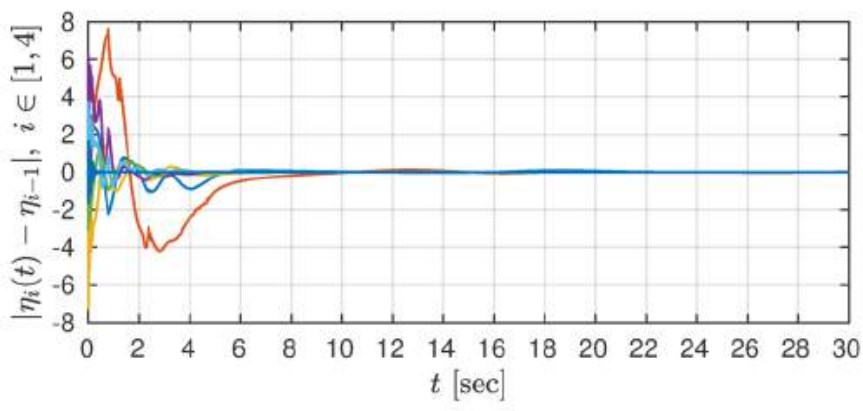

Fig. 3. Normed relative velocities for each pair leader-follower, with persistently exciting reference trajectories

The path followed by the vehicles in formation is depicted in Figure 4.

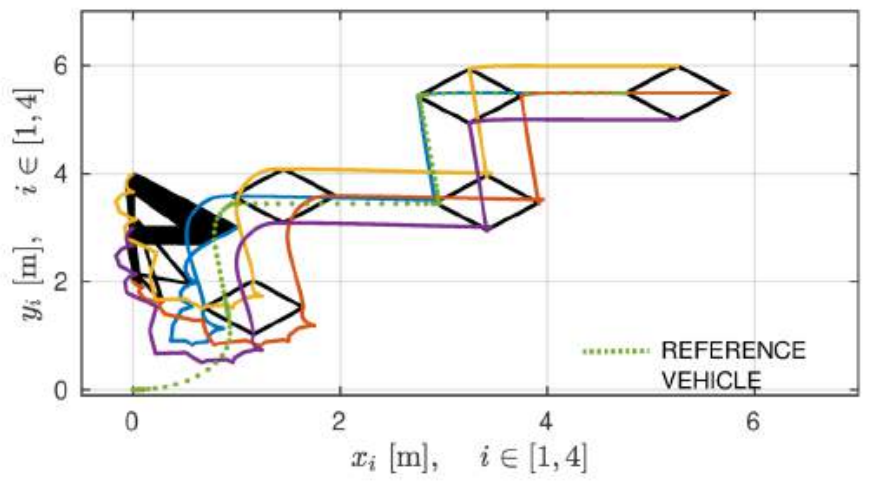

Fig. 4. Path followed by the formation with (truncated) persistently exciting trajectories.

Then, we use the adaptive velocity-tracking controller (24) with $\Theta:=\left[\begin{array}{lll}m_{1} & m_{2} & c\end{array}\right]^{\top}$ with adaptation gain $\gamma=10$. The initial values for $\hat{\Theta}$ are set to zero. In Figure 5 are depicted the norms of the parameter-estimation errors for each of the four vehicles, which converge to zero since the regressor evaluated along the reference trajectories,

$$
\Phi_{1}\left(t, \dot{\eta}_{r}, \eta_{r}, 0\right):=\left[\begin{array}{ccc}
\dot{v}_{r} & \dot{\omega}_{r} & \omega_{r}^{2} \\
\dot{\omega}_{r} & \dot{v}_{r} & -v_{r} \omega_{r}
\end{array}\right],
$$

is persisteodbdexitingtions test, the robots are required to follow a fictitious vehicle that slowly comes to a full-stop, that is, the reference velocities converge to zero with a "slow" convergence rate near zero; these are determined by 

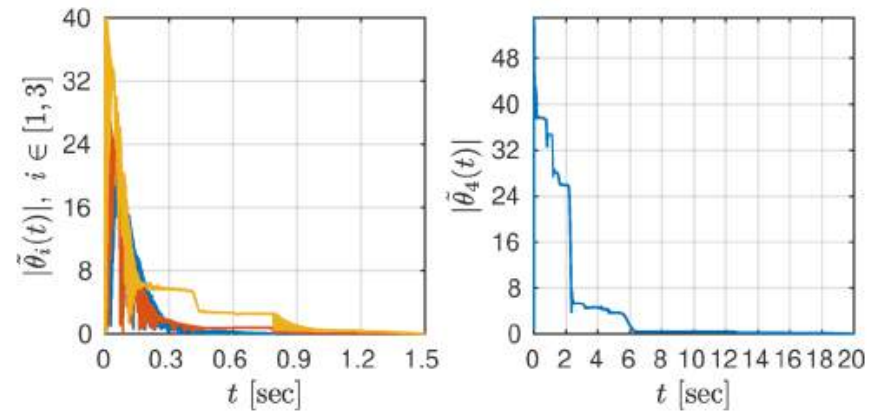

Fig. 5. Normed parameter estimation errors for each of the four vehicles

the solutions of $\dot{v}_{r}=-50 v_{r}^{3}$ and $\dot{\omega}_{r}=-100 \omega_{r}^{3}$ with initial conditions $v_{r}(0)=\omega_{r}(0)=1$. The corresponding position and velocity tracking errors, in norm, are showed in Figures 6 and 7 below.

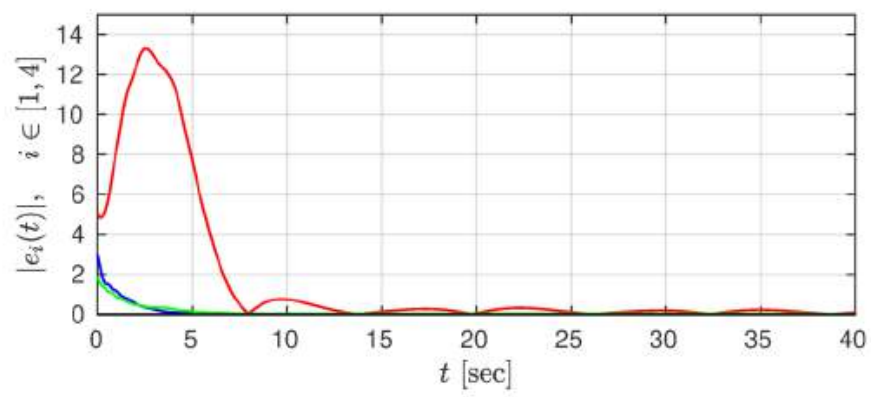

Fig. 6. Normed relative errors for each pair leader-follower, with vanishing reference trajectories

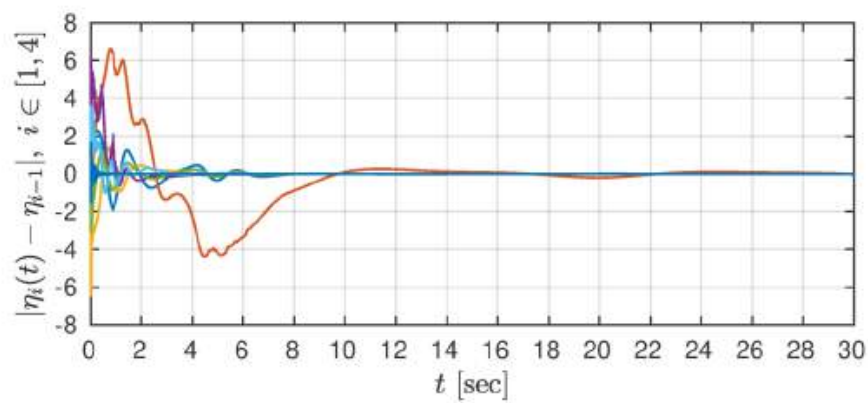

Fig. 7. Normed relative velocities for each pair leader-follower, with vanishing reference trajectories

\section{CONCLUSION}

We presented an adaptive controller for groups of autonomous vehicles following in formation persistentlyexciting, as well as converging reference trajectories. Our controller guarantees uniform asymptotic stabilisation and, in the case of persistently-exciting references, it also ensures the uniform convergence of the parameter estimation errors. Future work involves the formation control problem studied here with obstacle avoidance.

\section{REFERENCES}

[1] Y. Kanayama, Y. Kimura, F. Miyazaki, and T. Naguchi, "A stable tracking control scheme for an autonomous vehicle," in Proc. IEEE Conf. Robotics Automat., pp. 384-389, 1990.

[2] C. Samson, "Control of chained system: Application to path following and time-varying point stabilization of mobile robots," IEEE Trans. on Automatic Control, vol. 40, no. 1, pp. 64-77, 1995.

[3] K. D. Do, Z.-P. Jiang, and J. Pan, "A global output-feedback controller for simultaneous tracking and stabilization of unicycle-type mobile robots," IEEE Trans. on Robotics Automat., vol. 20, no. 3, pp. 589 594, 2004.

[4] J. Huang, C. Wen, W. Wang, and Z.-P. Jiang, "Adaptive output feedback tracking control of a nonholonomic mobile robot," Automatica, vol. 50 , no. 3 , pp. 821-831, 2014.

[5] K. S. Narendra and A. M. Annaswamy, Stable adaptive systems. New Jersey: Prentice-Hall, Inc., 1989.

6] C. C. de Wit, H. Khennouf, C. Samson, and O. J. Sørdalen, "Nonlinear control design for mobile robots", vol. 11 of Robotics and Automated Systems, ch. Recent Trends in Mobile Robots. Y. F. Zheng, ed., London: World Scientific, 1993.

[7] E. Panteley, E. Lefeber, A. Loria, and H. Nijmeijer, "Exponential tracking of a mobile car using a cascaded approach," in IFAC Workshop on Motion Control, (Grenoble, France), pp. 221-226, 1998.

[8] T. C. Lee, K. T. Song, C. H. Lee, and C. C. Teng, "Tracking control of unicycle-modeled mobile robots using a saturation feedback controller," IEEE Trans. Contr. Syst. Technol., vol. 9, pp. 305-318, Mar 2001.

[9] M. Maghenem, A. Loria and E. Panteley, "A robust $\delta$-persistently exciting controller for leader-follower tracking-agreement of multiple vehicles," European Journal of Control, vol. 40, pp. 1-12, 2018. Appeared online: sept. 2017. DOI: 10.1016/j.ejcon.2017,09.001.

[10] Y. Wang, Z. Miao, H. Zhong, and Q. Pan, "Simultaneous stabilization and tracking of nonholonomic mobile robots: A Lyapunov-based approach," IEEE Trans, on Control Systems Technology, vol. 23, pp. 1440-1450, July 2015.

[11] M. Maghenem, A. Loria, and E. Panteley, "A unique robust controller for tracking and stabilization of nonholonomic vehicles," Int. J. Control, 2018. In presss. DOI: 10.1080/00207179.2018.1554270.

[12] P. Morin and C. Samson, "Control of nonholonomic mobile robots based on the transverse function approach," IEEE Trans. on robotics, vol. 25 , no. 5 , pp. $1058-1073,2009$.

[13] W. E. Dixon, M. S. de Queiroz, D. M. Dawson, and T. J. Flynn, "Adaptive tracking and regulation of a wheeled mobile robot with controller/update law modularity," IEEE Trans. on control systems technology, vol. 12, no. 1, pp. 138-147, 2004.

[14] K. D. Do, Z.-P. Jiang, and J. Pan, "Simultaneous tracking and stabilization of mobile robots: an adaptive approach," IEEE Trans. on Automatic Control, vol. 49, no. 7, pp. 1147-1152, 2004.

[15] W. Wang, J. Huang, C. Wen, and H. Fan, "Distributed adaptive control for consensus tracking with application to formation control of nonholonomic mobile robots," Automatica, vol. 50, no. 4, pp. 1254 $1263,2014$.

[16] M. Maghenem, Stability and stabilization of networked time-varying systems. PhD thesis, Univ Paris Saclay, Gif sur Yvette, 2017.

[17] M. Maghenem, A. Loria, and E. Panteley, "Cascades-based leaderfollower formation-tracking and stabilization of multiple nonholonomic vehicles," IEEE Trans. on Automatic Control, 2018. In review.

[18] D. A. Lizárraga., "Obstructions to the existence of universal stabilizers for smooth control systems," Mathematics of Control, Signals and Systems, vol. 16, p. 255277, 2004.

[19] A. Loria, "From feedback to cascade-interconnected systems: Breaking the loop," in Proc. of the 47th. IEEE Conf. on Decision Control, (Cancun, Mex.), pp. 4109-4114, 2008.

[20] H. Khalil, Nonlinear systems. New York: Macmillan Publishing Co. 2nd ed., 1996.

[21] E. Panteley and A. Loria, "Growth rate conditions for stability of cascaded time-varying systems," Automatica, vol. 37, no. 3, pp. 453$460,2001$.

[22] A. Loria E. Panteley, D. Popovic, and A. Teel, " $\delta$-persistency of excitation: a necessary and sufficient condition for uniform attractivity," in Proc. of the 4lst. IEEE Conf. on Decision Control, (Las Vegas, CA. USA), pp. 3506-3511, 2002. Paper no. REG0623. 